\title{
Single Chamber Pacemaker
}

National Cancer Institute

\section{Source}

National Cancer Institute. Single Chamber Pacemaker. NCI Thesaurus. Code C80458.

A heart pacemaker with the capability of monitoring and stimulating either the atria or the ventricles when necessary. 